\title{
Retraction Note to: Involvement of a Specific Chemosensory Protein from Bactrocera dorsalis in Perceiving Host Plant Volatiles
}

\author{
Xin $\mathrm{Yi}^{1} \cdot$ PeiDan Wang ${ }^{1} \cdot$ Zheng Wang $^{1} \cdot \mathrm{Jun} \mathrm{Cai}^{1} \cdot$ MeiYing Hu ${ }^{1} \cdot$ GuoHua Zhong ${ }^{2}$
}

Published online: 7 June 2016

(C) Springer Science+Business Media New York 2016

Retraction to: J Chem Ecol (2014) 40:267-275

DOI 10.1007/s10886-014-0406-4

This article has been retracted by Xin Yi, PeiDan Wang, Zheng Wang, Jun Cai, MeiYing Hu, and GuoHua Zhong.

Reason: The authors have discovered a serious mistake in the paper. 3-Methylbutyl acetate was reported as binding to the protein. Upon further experimentation, it did not bind. The reason is unclear. After a reevaluation of this work, they no longer have confidence in the finding. Further validation is needed before a firm conclusion can be reached. The authors do not want to further mislead readers of this article.

The online version of the original article can be found at http://dx.doi.org/ 10.1007/s10886-014-0406-4.

\section{GuoHua Zhong}

guohuazhong@scau.edu.cn

1 Laboratory of Insect Toxicology, Key Laboratory of Pesticide and Chemical Biology, Ministry of Education, South China Agricultural University, Guangzhou, People's Republic of China

2 Key Laboratory of Pesticide and Chemical Biology, Ministry of Education, PR China, College of Natural Resources and Environment, South China Agricultural University,

Guangzhou 510642, China 